\title{
UCP1 variant and energy balance
}

A single nucleotide polymorphism in the promoter region of the gene encoding mitochondrial brown fat uncoupling protein 1 (UCP1) reduces thermogenesis in young women, according to Japanese investigators. Their findings highlight the importance of the metabolic actions of brown adipose tissue and the resultant effect on resting energy balance.

UCP1 plays a key part in adaptive thermogenesis. A polymorphism of UCP1 has been reported to be more frequent in Japanese than white populations. Narumi Nagai (University of Hyogo) and colleagues, therefore, set out to investigate in more detail whether a specific polymorphism (A/G) at position -3826 of the UCP1 gene influences the activity of the thermoregulatory sympathetic nervous system (SNS).

The investigators enrolled 82 healthy Japanese female volunteers aged 20-22 years. Participants fasted overnight before the study. UCP1-associated thermogenesis was determined using heart rate variability spectral analyses, whilst resting energy expenditure was measured by indirect calorimetry. Frequencies of the three UCP1 genotypes were 0.27 (A/A), $0.45(\mathrm{~A} / \mathrm{G})$ and $0.28(\mathrm{G} / \mathrm{G})$.

A reduction in resting energy expenditure, thermoregulatory SNS activity and dietary fat intake was reported in the G-allele carriers, which indicates that UCP1-linked thermogenesis is reduced in these women.

"The present results suggest that the attenuated UCP1-linked thermogenesis observed with the G/G allele of the UCP1 gene has an adverse effect on the regulation of whole-body energy balance, which requires consideration during treatment of obesity, especially in Asians," Nagai concludes.

\section{Rosanne Diaz}

Original article Nagai, N. et al. UCP1 genetic polymorphism (-3826 A/G) diminishes resting energy expenditure and thermoregulatory sympathetic nervous system activity in young females. Int. J. Obes. (Lond.) doi:10.1038/ ijo.2010.261 